\title{
The distribution of the gas phase in the bubble flow in the vertical assembly of $3 \times 3$ rods with gas injection from a single source
}

\author{
Maksim Vorobyev, Oleg Kashinsky, and Pavel Lobanov ${ }^{*}$ \\ Kutateladze Institute of Thermophysics, Novosibirsk, 630090, Acad. Lavrentiev ave., 1, Russia
}

\begin{abstract}
The work is devoted to an experimental study of the distribution of gas along the channel cross-section in the form of an assembly of vertical rods $3 \times 3$ in a square arrangement. The gas was introduced into the channel from the corner rod through a single capillary. Upstream, a local gas content sensor was attached to the the central fuel element simulator. The position of the sensor was changed by rotating the rod around its axis. The experiment was carried out at Reynolds numbers $\operatorname{Re}=4500-9000$ and flow rate gas contents of $2-4 \%$.
\end{abstract}

\section{Introduction}

In fuel assemblies of nuclear power plants, there is a possibility of boiling of the coolant on the surface of fuel elements. As a result of this process, a two-phase vapor-liquid flow occurs. In the literature there is a large number of works devoted to the investigation of gas and vapor-liquid flows in pipes. In particular, it is shown that with dispersed phase occurring the local structure of the flows is reconstructed, also the key thermohydraulic parameters, such as hydraulic resistance and heat transfer coefficients, can change significantly [1 - 6]. Due to the above, an actual task is to study the regularities of distribution of the disperse phase during the movement of two-phase flows in assemblies of rods and its effect on the thermal-hydraulic flow characteristics. The task of this work was to study the distribution of gas at a certain distance from a single source of the gas phase in the assembly of vertical rods $3 \mathrm{X} 3$ in a square arrangement.

\section{Experimental setup and experimental technique}

The experimental setup (Fig. 1) was a closed pipe filled with a fluid. Using the centrifugal pump (1), the liquid (distilled water with the addition of sodium carbonate) from the tank (2) was fed into a working channel of square section (3), which was an assembly of nine vertically oriented rods $9.1 \mathrm{~mm}$ in diameter. The geometry of the assembly model was fixed with using of spacing grid simulating the flow in the fuel assemblies of the reactor plants. A stainless steel capillary (4) was soldered into the one of the corner rods, through which gas (atmospheric air) using was supplied into the fluid flow. Gas flow rate was regulated by

*Corresponding author: lobanov@itp.nsc.ru 
Bronkhorst controller. The capillary was bent and directed along the fluid flow. The rod supplying gas was oriented in such a manner that the point of introduction of the gas into the flow was turned towards the centre of the channel. To the central simulator of fuel element was attached a sensor of local gas content (5), which is a platinum wire welded into a glass capillary. This detector was used in the mode of the conductivity sensor. An alternating voltage of $100 \mathrm{kHz}$ was applied to it. Its current increased and straightened. The low-frequency envelope of this signal had two levels corresponding to the detection of the sensor element in the liquid and gas phases. The signal of this sensor was used to determine the local gas content. The position of the sensor was changed by rotating the rod around its axis, which made it possible to obtain a diagram of the distribution of the local gas content along the circumference, the pitch was $15^{\circ}$. The flow rate was measured using a calibrated flow diaphragm (6). The pressure drop across the diaphragm was measured using a differential pressure gauge Metran-100 DD. The gas-liquid mixture from the outlet of the channel through a flexible tube was fed into the separation tank (7), where gas and liquid were separated, and then the liquid was merged into the main tank through a return pipe.
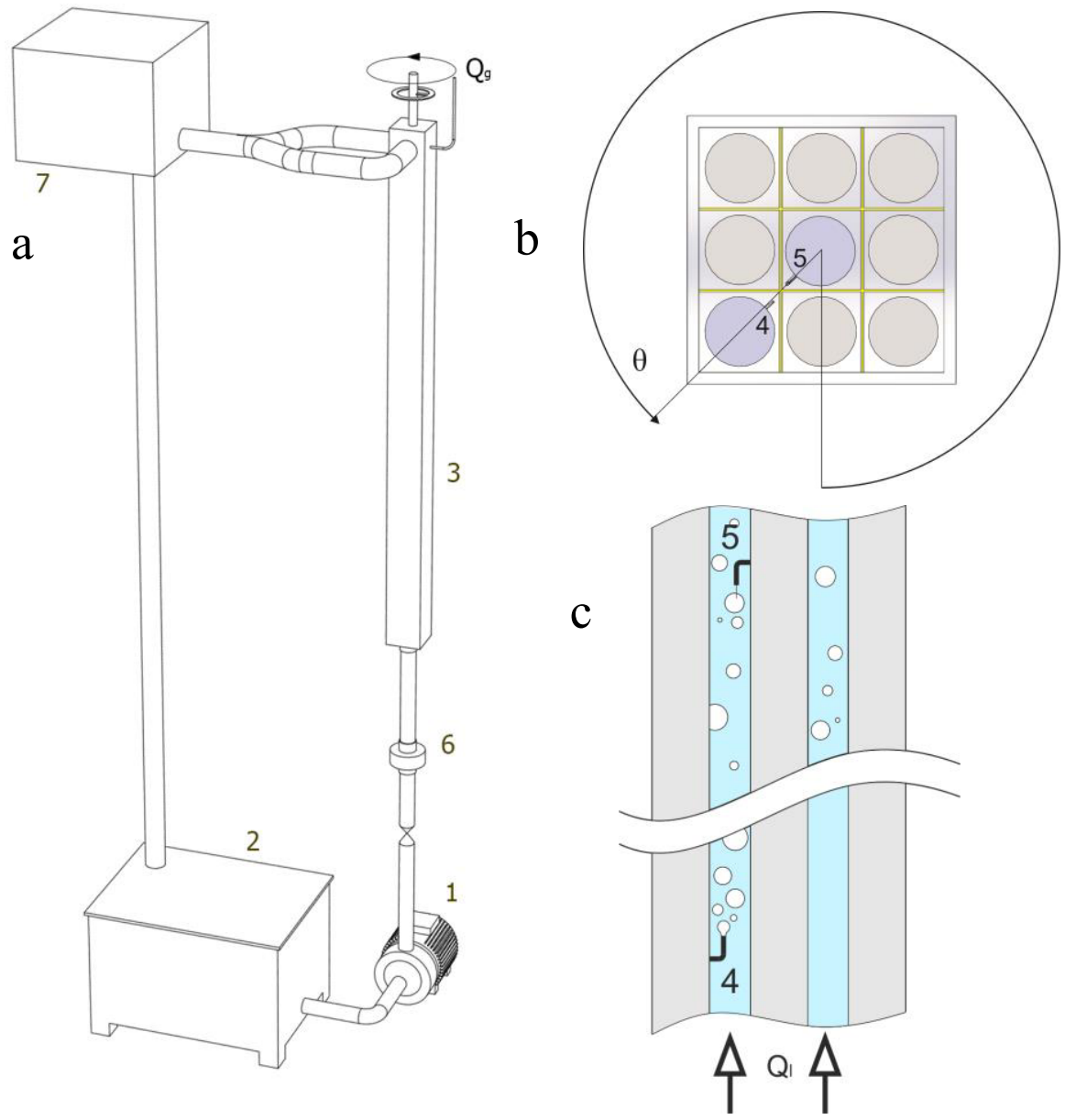

Fig. 1. (a) The scheme of the experimental setup; (b) Working channel top view; (c) Working channel side view. 


\section{Experimental results}

The experiment was carried out at Reynolds numbers $\mathrm{Re}=4500-9000$ and gas flow rates from $500-1000 \mathrm{ml} / \mathrm{min}$, which corresponded to gas content from $2 \%$ to $4 \%$. The hydraulic diameter, included in the Reynolds number, was defined as the quadruple ratio of the channel area to its perimeter.

Distributions of local gas content around the central fuel element were obtained at different Reynolds numbers and gas consumption rates. The measuring section was located above the spacing grid. In Fig. $2 \mathrm{a}$ the dependences for $\mathrm{Re}=4500-9000$ and gas flow rate of $1000 \mathrm{ml} / \mathrm{min}$ are presented. In Fig. $2 \mathrm{~b}$ the results are presented for a constant liquid flow rate $(\mathrm{Re}=4500)$, but at different gas flow rates.

One can see that there is a significant uneven distribution of the local gas content. The presence of the spacing grid leads to an increased diffusion of the gas phase along the channel cross-section.

a
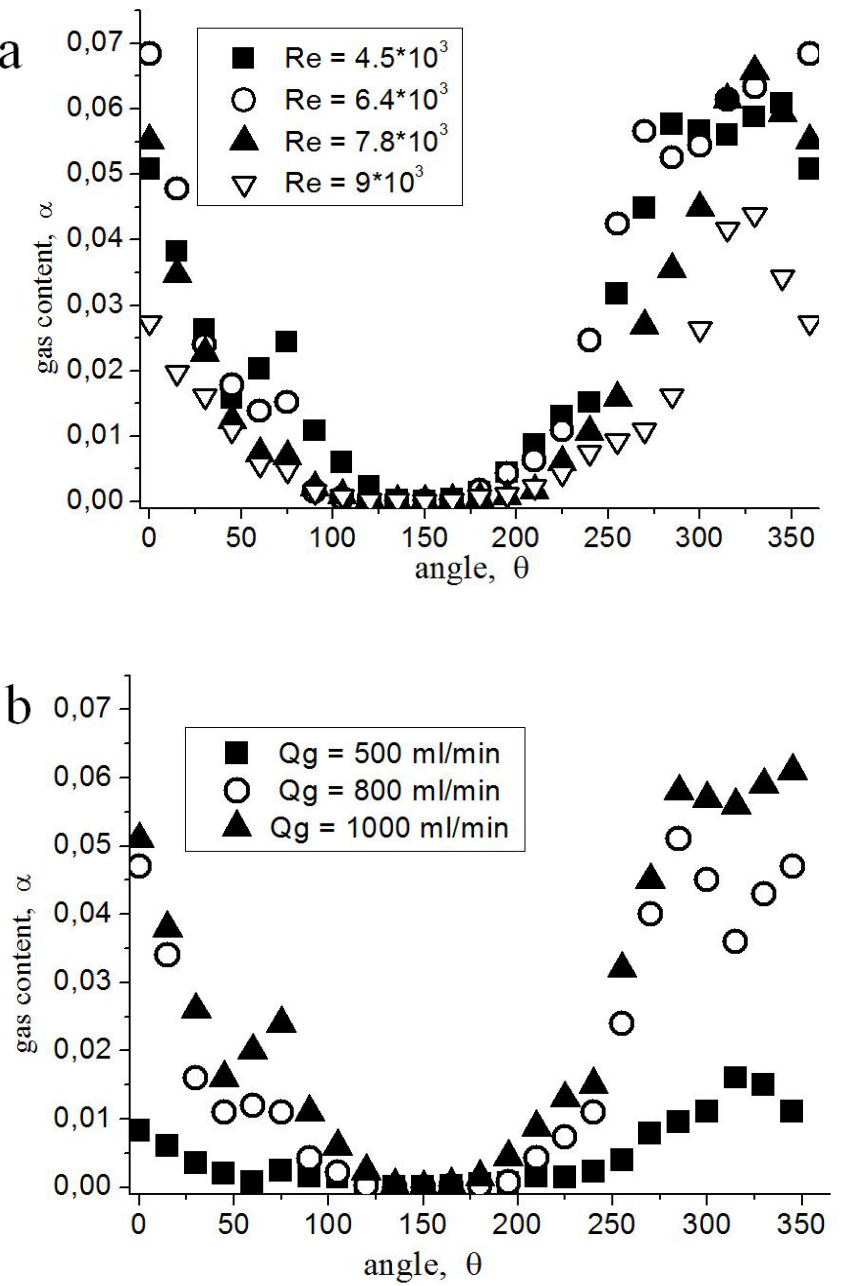

Fig. 2. Dependence of the local gas content on the angle of rotation of the sensor; (a) different Reynolds numbers, $\mathrm{Q}_{\mathrm{g}}=1000 \mathrm{ml} / \mathrm{min}$; (b) different gas flow rates, $\mathrm{Re}=4500$. 


\section{References}

1. V.E. Nakoryakov, O.N. Kashinsky, A.P. Burdukov, V.P. Odnoral. Int. J. Multiphase Flow 7, 63 (1981)

2. S.K. Wang, S.J. Lee, O.S. Jones Jr., R.T. Lahey Jr., Int. J. Multiphase Flow 13, 327 (1987)

3. M.A. Pakhomov, V.I. Terekhov, Int. J. Heat Mass Transfer 101, 1251 (2016)

4. D. Métrailler, S. Reboux, D. Lakehal, Nuclear Eng. Design (2017) (to be published)

5. J.P. Schlegel, S. Sharma, R.M. Cuenca, T. Hibiki, M. Ishii, Int. J. Heat Fluid Flow 47, 42 (2014)

6. S. Dabiri, G. Tryggvason, Chem. Eng. Sci. 122, 106 (2015) 